\title{
Dcf1 regulates neuropeptide expression and maintains energy balance
}

\author{
Qiang Liu†, Yu Chen†, Qian Li, Liang Wu, Tieqiao Wen*
}

Laboratory of molecular neural biology, School of life sciences, Shanghai

University, 333 Nanchen Road, Shanghai, 200444;

$\dagger:$ These authors contributed equally to this work

*: To whom correspondence may be addressed

E-mail: wtq@shu.edu.cn; Tel/Fax: 86-021-66132512

Keywords: Dcf1; NPY; NPY-Y1; GABA; energy balance 


\section{Abstract}

Neuropeptide $\mathrm{Y}$ (NPY) is an important neurotransmitter in the brain that plays a pivotal role in food intake and energy storage. Although many studies have focused on these functions, the regulation of NPY expression remains unclear. Here we showed that dendritic cell factor 1 (Dcf1) regulates NPY expression and maintains energy balance. We found that NPY expression is significantly reduced in the hypothalamus of Dcf1 knockout (Dcf1-/-, KO) mice. In contrast, Dcf1 overexpression significantly increases NPY expression in the cell line. We also found that Dcf1 acts upstream of the NPY gene to regulate NPY expression and modulates the NPY-NPY receptor 1-GABA signal. Notably, we observed a significant increase in the ATP concentration in Dcf1-l- mice, suggesting a greater demand for energy in the absence of Dcf1. We studied the relationship between Dcf1 and NPY and revealed that Dcf1 plays a critical role in energy balance. 


\section{Introduction}

Energy balance is essential for maintaining activities of daily living, and its imbalance will inevitably lead to disease. Genetic studies have led to the discovery of key regulators of energy balance [5], such as neuropeptide $Y$ (NPY) [19] and melanocortin peptides encoded by the pro-opiomelanocortin (POMC) gene [6]. In addition, the reciprocal interaction between POMC and NPY/agouti-related peptide neurons has been confirmed [7, 13, 20].

NPY and NPY receptors are widely expressed in the mammalian central nervous system [2]. NPY is generally believed to enhance appetite and promote adipose tissue growth [15], while a loss of the NPY gene increases susceptibility to stress and anxiety [9, 23]. NPY also acts as an endogenous modulator of neuronal activity to affect GABAergic and glutamatergic transmission [22]; therefore, the release of NPY/GABA facilitates the maturation of neuronal synapses [21]. Mechanistically, NPY functions through several G-protein-coupled receptors (GPCRs) [12]. Of these, the NPY receptor 1 (NPY-Y1) is the most important for energy balance $[1,18]$. NPY-Y1 gene knockout $(\mathrm{KO})$ in mice causes a dramatic increase in food intake and a decrease in physical activity or energy expenditure, eventually leading to obesity $[1,12]$.

Dendritic cell factor 1 (Dcf1) is a single transmembrane protein that is abundantly expressed in the nervous system. In our previous study, we found 
that Dcf1 is involved in neural stem cell differentiation [25]; therefore, we constructed a Dcf1 KO mouse to explore its impact on neural development. We observed a significant decrease in the dendritic spine density, spine head size, dendritic arborization, and postsynaptic density length in hippocampal synapses of Dcf1-I- mice [11]. In this study, we used Dcf1 KO mice to examine the role of Dcf1 in food intake, NPY regulation, and maintenance of energy balance.

\section{Material and methods}

\section{Animals}

All experiments were conducted on male C57BL/6J mice. Mice were housed under a controlled temperature $\left(23 \pm 1^{\circ} \mathrm{C}\right)$ on a 12-h light-dark cycle (08:00-20:00) with food and water provided. The body weight and daily food intake were recorded for 12 weeks after birth. Tissue samples for protein extraction and immunohistochemical observations were obtained from four to five mice per strain. All animals used for the experiment were approved by the Shanghai University Ethics Committee.

\section{Western blotting}

Extraction of proteins from cells: 293T cells were transfected in 12-well plates with PCAGGS-EGFP, PCAGGS-Dcf1-EGFP, and PCAGGS-NPY-EGFP $(2 \mu \mathrm{g})$ 
recombinant plasmids. Cells were lysed at $48 \mathrm{~h}$ post-transfection, and lysates were blotted for the indicated antibodies.

Extraction of proteins from tissues: Hypothalamic and hippocampal tissues obtained from 1- and 3-month-old Dcf1 $1^{+/+}$and Dcf1 ${ }^{-/-}$mice $(n=4$ per strain at each time point) were individually homogenized in lysis buffer (Beyotime) containing protease and phosphatase inhibitors.

Protein concentrations were determined using the BCA Protein Assay Kit (Beyotime). Total protein was separated using $12 \%$ or $15 \%$ SDS-PAGE and electrophoretically transferred onto polyvinylidene difluoride membranes (EMD Millipore, Billerica, MA, USA), which were blocked with 5\% BSA-PBS for $1 \mathrm{~h}$ at room temperature and incubated overnight at $4^{\circ} \mathrm{C}$ with one of the following primary antibodies in $0.1 \%$ PBST: rabbit polyclonal anti-NPY (1:1000, Abcam), anti-NPY-Y1 (1:1500, Novus Biologicals), anti-GAPDH antibody (1:1000, Santa Cruz), and anti-Dcf1 (1:3000, produced in-house). Next, infrared dye 800-conjugated affinity-purified goat anti-mouse $\lg G$ and infrared dye 700-labeled goat anti-rabbit IgG (Zemed, USA) secondary antibodies were added and incubated for 30 min at room temperature. Immunolabeled bands were detected and quantified using the LI-COR Odyssey Infrared Imaging System (simultaneous two-color targeted analysis).

\section{Immunofluorescence}


Brain samples obtained from Dcf1 ${ }^{+/+}$(wild type, WT) and Dcf1 ${ }^{-/}$mice at 3 months of age $(n=5)$ were fixed in $4 \%$ paraformaldehyde for $24 \mathrm{~h}$ at $4^{\circ} \mathrm{C}$, dehydrated, embedded, frozen, and cut into thick coronal sections of $20 \mu \mathrm{m}$ thickness. These sections were washed in PBS thrice (5 min per wash), permeabilized with $0.1 \%$ Triton $\mathrm{X}-100$ for $30 \mathrm{~min}$, and blocked using $2 \% \mathrm{BSA}$ for $60 \mathrm{~min}$ at room temperature. The sections were then incubated with primary antibodies overnight at $4^{\circ} \mathrm{C}$. After washing thrice with PBS, the sections were incubated with secondary antibodies for $2 \mathrm{~h}$ and stained with the fluorescent dye DAPI for $10 \mathrm{~min}$ at room temperature. The fluorescence emission at 488 $\mathrm{nm}$ (FITC) and $543 \mathrm{~nm}$ (TRITC) wavelengths was detected by confocal microscopy.

\section{ATP assay}

The ATP concentration was detected using the ATP Assay Kit (Beyotime). In brief, 293T cells or tissues were lysed and lysates were centrifuged at 12,000 rpm for $10 \mathrm{~min}$ to isolate total protein. The supernatant was added to the ATP detection solution at a 1:1 ratio, and the standard samples were generated at the same time. Luminescence was immediately detected using a plate reader (PerkinElmer). The protein concentration was measured using the Bradford assay. For the ATP stimulation experiment, 293T cells were primed incubated with LPS $(1 \mu \mathrm{g} / \mathrm{ml})$ for $4 \mathrm{~h}$ and subsequently stimulated for 30 min with $3 \mathrm{mM}$ ATP. 


\section{Statistics}

All data are expressed as mean \pm SEM. Student's t-test was used to compare data between two groups, and one-way ANOVA, followed by an appropriate post hoc test, was used to compare data among multiple groups.

\section{Results}

\section{Dcf1 regulates food intake through NPY}

Food intake was significantly lower in Dcf1 $1^{-/}$mice than in Dcf1 $1^{+/+}$mice (Fig. 1A), suggesting an important role of Dcf1 in maintaining appetite. NPY and POMC are central regulators of food intake and weight. NPY expression in the hypothalamus of Dcf1-/- mice was detected by western blotting. The results revealed a significant reduction in NPY expression in Dcf1 ${ }^{-/}$mice at 1 month and 3 months (Fig. 1B, 1C); however, no significant change was observed in the hippocampus at these two stages (Fig. 1B, 1D). In addition, there was no significant change in the POMC expression level in the hypothalamus (Fig. 1B, 1E) or hippocampus (Fig. 1B, 1F). These results suggest that Dcf1 regulates NPY but not POMC in the hypothalamus.

\section{Dcf1 regulates NPY expression}

Furthermore, we examined NPY expression at different locations within the hypothalamus in detail by immunofluorescence. We found that compared with WT mice, NPY expression was reduced in the paraventricular nucleus (PVN), 
dorsomedial hypothalamus (DMH), and arcuate nucleus (ARC) of Dcf1-/- mice (Fig. 2A-2F). These results were consistent with western blot results. To distinguish the regulatory relationship between Dcf1 and NPY, we overexpressed them in 293T cells. The results revealed that compared with WT controls, Dcf1 overexpression significantly increased NPY expression (Fig. 2G, 2H); however, NPY overexpression did not alter Dcf1 expression (Fig. 2G, 21). This indicated that Dcf1 regulates NPY expression.

NPY-Y1 presents a significant reduction with the decrease of NPY in KO mice Numerous studies have suggested that downstream of the NPY-Y1 receptor, NPY regulates a class A of GPCRs, which is expressed in both neuronal and non-neuronal tissues [4]. In view of these results, we examined NPY-Y1 expression in the hypothalamus of WT and KO mice at 1 month and 3 months. The results showed a significant decrease in NPY-Y1 expression in KO mice at 3 months, but not at 1 month (Fig. 3A, 3B). Further, we examined NPY-Y1 expression in the hypothalamus at 3 months by immunofluorescence staining and found a notable decrease in the expression in DMH and ARC areas, whereas, WT mice showed strong NPY-Y1 expression in these areas (a high expression of NPY-Y1 was observed in these two areas) (Fig. 3C-3F). We also examined whether Dcf1 overexpression in 293T cells could alter NPY-Y1 expression and found that Dcf1 overexpression significantly increased NPY-Y1 expression compared with that in WT controls (Fig. 3G, 3H), indicating that Dcf1 regulates the NPY-NPY-Y1 system. 


\section{Dcf1 affects GABA through NPY}

Considering the important role of the NPY/GABA system in brain function, we examined GABA expression at 1 month and 3 months. Compared with WT controls, western blotting revealed a significant reduction in GAD67 in the hypothalamus of Dcf1-/- mice at 3 months (Fig. 4A, 4C) but not at 1 month (Fig. 4A, 4B), suggesting a reduction in the hypothalamic GABA at 3 months. To further explore the relationship between Dcf1 and GABA, we generated Dcf1-/GAD-EGFP mice by crossing Dcf1-/- mice with GAD-EGFP reporter mice expressing green fluorescent protein (GFP) in GABA neurons to study the neuronal activity. The results indicated significantly lower fluorescence values in the hypothalamus of Dcf1 ${ }^{-/-}$mice than in that of WT controls at 3 months (Fig. 4D, 4E), indicating a regulation of Dcf1 on GABA neurons in the hypothalamus.

\section{Dcf1 KO elevates ATP concentration}

A previous study suggested that fasting-induced systemic ghrelin increases glucose levels and reduces ATP hydrolysis and ATP levels, particularly in ARC [8]. The ATP concentration in the hypothalamus was evaluated. Compared with WT controls, the ATP concentration was found to be significantly increased in the hypothalamus of Dcf1-/- mice at 3 months but no change was observed at 1 month (Fig. 5A). In contrast, Dcf1 and NPY overexpression in 293T cells caused a significant decrease compared with that in WT controls (Fig. 5B). These results indicated that Dcf1 deficiency increases energy 
consumption. Further, by increasing the ATP level in 293T cells, we detected that both Dcf1 and NPY levels were reduced, although the difference was not significant (Fig. 5C-5E), which suggested that ATP engages in the signaling mechanism for food intake mediated by Dcf1-NPY.

\section{Discussion}

We proposed that the energy balance regulated by Dcf1 is an internalization-independent pathway mediated via NPY and NPY-Y1. We demonstrated that NPY expression is controlled by Dcf1, which provides a new insight on NPY regulation. Moreover, surprisingly, we observed that the ATP concentration is increased in the hypothalamus of Dcf1-/- mice. This unexpected reciprocal relationship between Dcf1 expression and ATP concentration was confirmed at the tissue and cellular level by Dcf1 KO and by overexpression of Dcf1 or NPY in 293T cells. A possible explanation for this relationship is that Dcf1 plays a role in promoting ATP synthesis or reducing ATP consumption and Dcf1-/- mice require more energy to maintain activities of daily living.

ARC plays an important role in the hypothalamic control of energy balance, which is involved in the regulation of food intake by NPY, POMC, and tyrosine hydroxylase (TH) [17]. DMH lesions impair food-entrainable circadian rhythms $[3,14]$. GABAergic neurons, including those in $\mathrm{DMH}$, could be the principal orexigenic neurons targeted by leptin [24]. Further, the optical activation of 
axonal terminals of DMH GABAergic neurons in the PVN, where anorexigenic neurons are localized, led to an increase in inhibitory postsynaptic currents on PVN neurons and promoted food intake [16].

The activation and reduction of NPY systems may be the factors that are involved in triggering rebound hyperphagia [10]. However, the upstream control mechanisms were unclear. Here we revealed that Dcf1 acts upstream of the NPY gene to regulate NPY expression and modulates the NPY-NPY receptor 1-GABA signal. In summary, this study highlights the role of Dcf1 in energy balance and provides a new foundation for further studies on the mechanism of energy balance.

\section{Acknowledgments}

This work was funded by the National Science Foundation of China (81271253, 81471162), the Science and Technology Commission of Shanghai (14JC1402400), and the Key Innovation Project of Shanghai Municipal Education Commission (Grant No.14ZZ090).

\section{Conflict of interest}

The authors declare no conflicts of interest. 


\section{References}

[1] I. Bertocchi, A. Oberto, A. Longo, P. Mele, M. Sabetta, A. Bartolomucci, P. Palanza, R. Sprengel, C. Eva, Regulatory functions of limbic Y1 receptors in body weight and anxiety uncovered by conditional knockout and maternal care, Proceedings of the National Academy of Sciences of the United States of America 108 (2011) 19395-19400.

[2] J. Duarte-Neves, L. Pereira de Almeida, C. Cavadas, Neuropeptide Y (NPY) as a therapeutic target for neurodegenerative diseases, Neurobiology of disease 95 (2016) 210-224.

[3] J.J. Gooley, A. Schomer, C.B. Saper, The dorsomedial hypothalamic nucleus is critical for the expression of food-entrainable circadian rhythms, Nature neuroscience 9 (2006) 398-407.

[4] H. Herzog, M. Baumgartner, C. Vivero, L.A. Selbie, B. Auer, J. Shine, Genomic organization, localization, and allelic differences in the gene for the human neuropeptide Y Y1 receptor, The Journal of biological chemistry 268 (1993) 6703-6707.

[5] D. Hirsch, Z. Zukowska, NPY and stress 30 years later: the peripheral view, Cellular and molecular neurobiology 32 (2012) 645-659.

[6] P.A. Jimenez-Vasquez, A.A. Mathe, J.D. Thomas, E.P. Riley, C.L. Ehlers, Early maternal separation alters neuropeptide $Y$ concentrations in selected brain regions in adult rats, Brain research. Developmental brain research 131 (2001) 149-152.

[7] S.J. Kim, S.J. Lee, S. Lee, S. Chae, M.D. Han, W. Mar, K.W. Nam, Rutecarpine ameliorates bodyweight gain through the inhibition of orexigenic neuropeptides NPY and AgRP in mice, Biochemical and biophysical research communications 389 (2009) 437-442.

[8] H. Kurita, K.Y. Xu, Y. Maejima, M. Nakata, K. Dezaki, P. Santoso, Y. Yang, T. Arai, D. Gantulga, S. Muroya, A.K. Lefor, M. Kakei, E. Watanabe, T. Yada, Arcuate $\mathrm{Na}+, \mathrm{K}+-\mathrm{ATPase}$ senses systemic energy states and regulates feeding behavior through glucose-inhibited neurons, American journal of physiology.

Endocrinology and metabolism 309 (2015) E320-333.

[9] G. Lach, T.C. de Lima, Role of NPY Y1 receptor on acquisition, consolidation and extinction on contextual fear conditioning: dissociation between anxiety, locomotion and non-emotional memory behavior, Neurobiology of learning and memory 103 (2013) 26-33.

[10] N. Lauzurica, L. Garcia-Garcia, S. Pinto, J.A. Fuentes, M. Delgado, Changes in NPY and POMC, but not serotonin transporter, following a restricted feeding/repletion protocol in rats, Brain research 1313 (2010) 103-112.

[11] Q. Liu, Feng, R., Chen, Y. et al., Dcf1 Triggers Dendritic Spine Formation and Facilitates Memory Acquisition, Mol Neurobiol (2017).

[12] A. Longo, P. Mele, I. Bertocchi, A. Oberto, A. Bachmann, A. Bartolomucci, P. Palanza, R. Sprengel, C. Eva, Conditional inactivation of neuropeptide Y Y1 receptors unravels the role of $\mathrm{Y} 1$ and $\mathrm{Y} 5$ receptors coexpressing neurons in anxiety, Biological psychiatry 76 (2014) 840-849.

[13] A.J. Mercer, S.T. Hentges, C.K. Meshul, M.J. Low, Unraveling the central proopiomelanocortin neural circuits, Frontiers in neuroscience 7 (2013) 19.

[14] M. Mieda, S.C. Williams, J.A. Richardson, K. Tanaka, M. Yanagisawa, The dorsomedial hypothalamic nucleus as a putative food-entrainable circadian pacemaker, Proceedings of the National Academy of Sciences of the United States of America 103 (2006) 12150-12155. 
[15] N. Nara-ashizawa, T. Tsukada, K. Maruyama, Y. Akiyama, N. Kajimura, K. Yamaguchi, Response of hypothalamic NPY mRNAs to a negative energy balance is less sensitive in cachectic mice bearing human tumor cells, Nutrition and cancer 41 (2001) 111-118.

[16] Z. Otgon-Uul, S. Suyama, H. Onodera, T. Yada, Optogenetic activation of leptinand glucose-regulated GABAergic neurons in dorsomedial hypothalamus promotes food intake via inhibitory synaptic transmission to paraventricular nucleus of hypothalamus, Molecular metabolism 5 (2016) 709-715.

[17] E. Pollatzek, N. Hitzel, D. Ott, K. Raisl, B. Reuter, R. Gerstberger, Functional expression of $\mathrm{P} 2$ purinoceptors in a primary neuroglial cell culture of the rat arcuate nucleus, Neuroscience 327 (2016) 95-114.

[18] N. Pronchuk, A.G. Beck-Sickinger, W.F. Colmers, Multiple NPY receptors Inhibit $\operatorname{GABA}(\mathrm{A})$ synaptic responses of rat medial parvocellular effector neurons in the hypothalamic paraventricular nucleus, Endocrinology 143 (2002) 535-543.

[19] R. Sah, T.D. Geracioti, Neuropeptide $Y$ and posttraumatic stress disorder, Molecular psychiatry 18 (2013) 646-655.

[20] M.M. Scott, Y. Xu, C.F. Elias, K.W. Williams, Central regulation of food intake, body weight, energy expenditure, and glucose homeostasis, Frontiers in neuroscience 8 (2014) 384.

[21] X. Sun, T. Fukami, T. Li, M. Desai, M.G. Ross, Preferential development of neuropeptide Y/GABA circuit in hypothalamic arcuate nucleus in postnatal rats, Brain research 1635 (2016) 27-40.

[22] J. Veliskova, D. Iacobas, S. Iacobas, G. Sidyelyeva, T. Chachua, L. Velisek, Oestradiol Regulates Neuropeptide Y Release and Gene Coupling with the GABAergic and Glutamatergic Synapses in the Adult Female Rat Dentate Gyrus, Journal of neuroendocrinology 27 (2015) 911-920.

[23] D. Verma, R.O. Tasan, H. Herzog, G. Sperk, NPY controls fear conditioning and fear extinction by combined action on $Y(1)$ and $Y(2)$ receptors, British journal of pharmacology 166 (2012) 1461-1473.

[24] L. Vong, C. Ye, Z. Yang, B. Choi, S. Chua, Jr., B.B. Lowell, Leptin action on GABAergic neurons prevents obesity and reduces inhibitory tone to POMC neurons, Neuron 71 (2011) 142-154.

[25] T. Wen, P. Gu, F. Chen, Discovery of two novel functional genes from differentiation of neural stem cells in the striatum of the fetal rat, Neuroscience letters 329 (2002) 101-105. 


\section{Figure legends}

Fig. 1 Dcf1 regulates food intake through NPY. (A) Monitoring for 3 months after birth showed reduced food intake in Dcf1 ${ }^{-/-}$mice. Two-tailed t-test, ${ }^{*} \mathrm{p}<$ $0.05,{ }^{* *} p<0.01,{ }^{* * *} p<0.001$; all data are presented as mean $\pm \mathrm{SEM} ; 10$ mice per group. (B) NPY and POMC levels in the hypothalamus and hippocampus were quantified by western blotting at 1 month and 3 months. (C, D) Statistical analysis of NPY expression in the hypothalamus and hippocampus at 1 month and 3 months. (E, F) Statistical analysis of POMC in the hypothalamus and hippocampus at 1 month and 3 months. Two-tailed t-test, ${ }^{*} p<0.05,{ }^{* *} p<0.01$, ${ }^{* * *} p<0.001$; all data are presented as mean \pm SEM; four to five mice per group.

Fig. 2 Dcf1 regulates NPY expression. Dcf1-/- mice exhibited significantly reduced NPY expression in PVN (A), DMH (C), and ARC (E). Corresponding statistical analyses are shown in (B), (D), and (F), respectively. Scale bar: 200 $\mu \mathrm{m}$, two-tailed t-test, ${ }^{*} \mathrm{p}<0.05,{ }^{* *} \mathrm{p}<0.01,{ }^{* * *} \mathrm{p}<0.001$; all data are presented as mean \pm SEM; four to five mice per group. (G) NPY expression significantly increased in cells transfected with Dcf1 compared with that in WT controls, whereas Dcf1 expression did not significantly change in cells transfected with NPY compared with that in WT controls. $(\mathrm{H}, \mathrm{I})$ Statistical analyses of NPY and Dcf1 expression. One-way ANOVA, ${ }^{*} p<0.05,{ }^{* *} p<0.01,{ }^{* * *} p<0.001$; all data are presented as mean \pm SEM, three samples per group. 
Fig. 3 Dcf1 knockout changes NPY-Y1 expression. (A) Western blot showing downregulation of NPY-Y1 expression in the hypothalamus of Dcf1 $1^{-1-}$ mice at 3 months. (B) Statistical analysis indicated a significant difference at 3 months. (C-F) Immunfluorescence staining showed that Dcf1 knockout reduces NPY-Y1 expression in DMH (red box) (C, D) and ARC (E, F). Statistical analysis indicated a significant difference (D) and (F). Scale bar: $200 \mu \mathrm{m}$, two-tailed t-test, ${ }^{*} p<0.05,{ }^{* *} p<0.01,{ }^{* * *} p<0.001$; all data are presented as mean \pm SEM; four to five mice per group. $(G, H)$ Overexpression of Dcf1 could increase the level of NPY-Y1. Two-tailed t-test, ${ }^{*} p<0.05,{ }^{* *} p<0.01,{ }^{* * *} p<$ 0.001 ; all data presented as mean $\pm \mathrm{SEM}$; three samples per group.

Fig. 4 Dcf1 knockout reduces GABA neurons. (A) Western blot of GAD67 in the hypothalamus of Dcf1- $1^{--}$mice at 1 month and 3 months. (B) Statistical analysis showed that GAD67 was not changed in the hypothalamus of Dcf1 $1^{-1}$ mice at 1 month. (C) Statistical analysis showed that GAD67 was downregulated in the hypothalamus of $\mathrm{Dcf}^{-1-}$ mice at 3 months. Two -tailed t-test, ${ }^{*} p<0.05,{ }^{* *} p<0.01,{ }^{* * *} p<0.001$; all data are presented as mean \pm SEM; three mice per group. (D, E) Dcf1 knockout reduces GABA neurons in the hypothalamus of GAD67-GFP mice; Scale bar: $200 \mu \mathrm{m}$. Two-tailed t-test, ${ }^{*} p<0.05,{ }^{* *} p<0.01,{ }^{* * *} p<0.001$; all data are presented as mean \pm SEM; four to five mice per group.

Fig. 5 Dcf1 knockout enhances ATP concentration. (A) Compared with Dcf1+/+ 
mice, Dcf1 ${ }^{-1}$ mice showed significantly increased ATP concentration in the hypothalamus at 3 months but no significant change at 1 month Two-tailed t-test, ${ }^{*} \mathrm{p}<0.05,{ }^{* *} \mathrm{p}<0.01,{ }^{* * *} \mathrm{p}<0.001$; all data presented as mean \pm SEM; four to five mice per group. (B) Dcf1 or NPY overexpression in 293T cells significantly reduced ATP concentration compared with that in WT controls. (C-E) Increasing the ATP level in 293T cells, both Dcf1 and NPY levels were reduced, although the difference was not significant. Two-tailed t-test, all data are presented as mean \pm SEM, three samples per group. 
A $B$

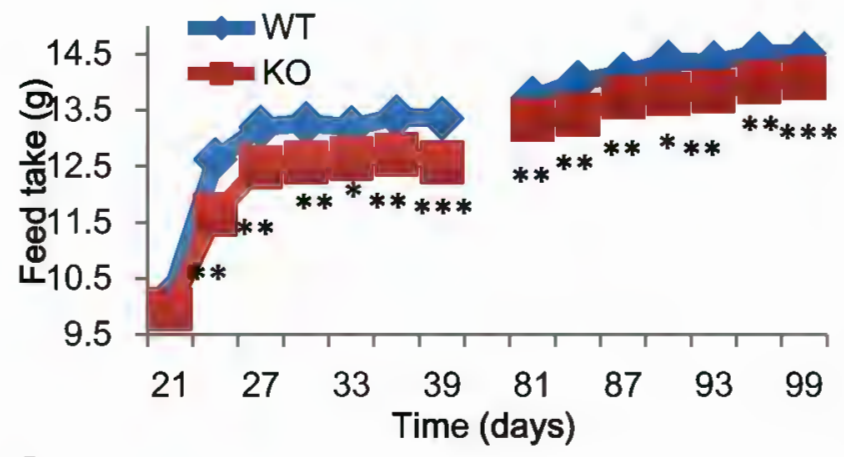

Hypothalamus NPY

Hippocampus NPY

One Three $\frac{\text { month }}{\mathrm{WT} \quad \mathrm{KO}} \frac{\text { months }}{\mathrm{WT} \mathrm{KO}}$

C

Hypothalamus NPY

Hypothalamus POMC

Hippocampus POMC

GAPDH

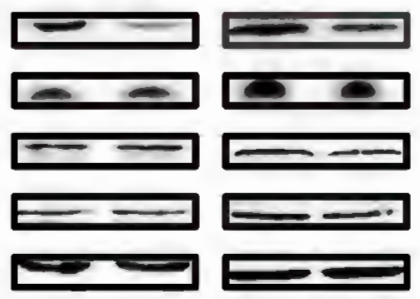

D

Hippocampus NPY

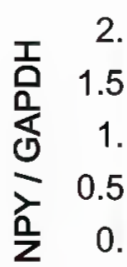

$E$

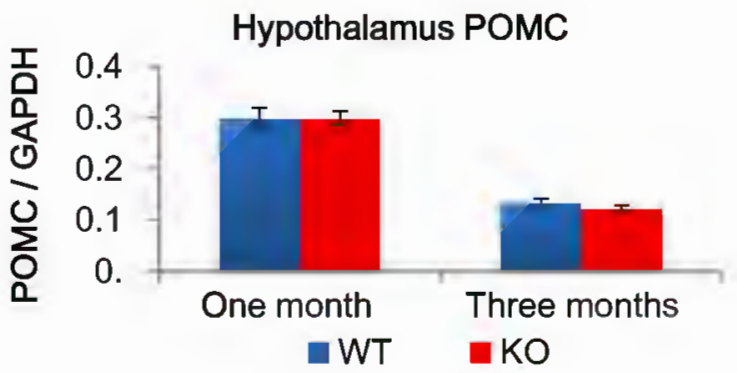

One month Three months

WT $\quad \mathrm{KO}$

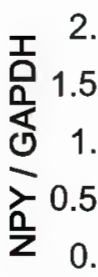

F

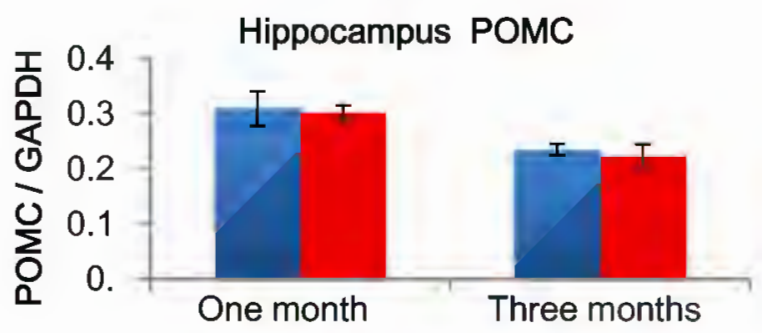

-WT $\quad \mathrm{KO}$ 
A

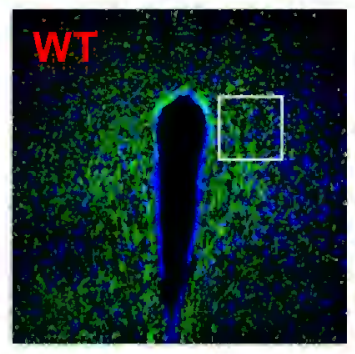

C

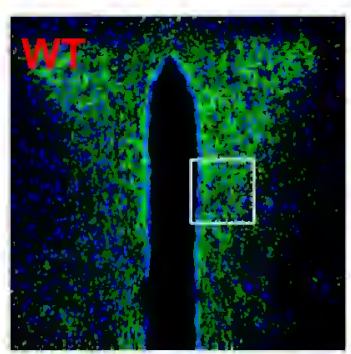

E

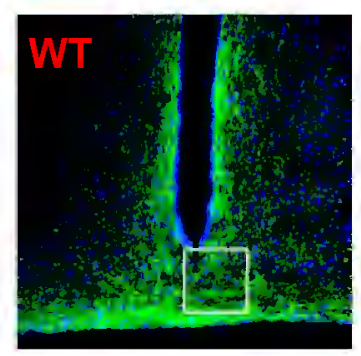

G

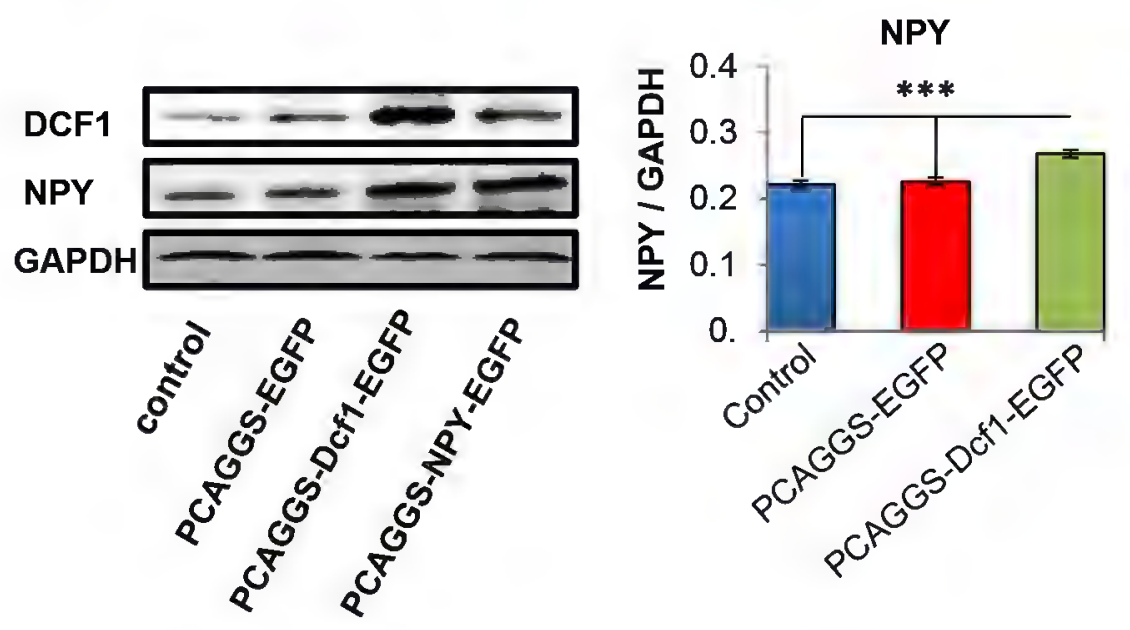

B

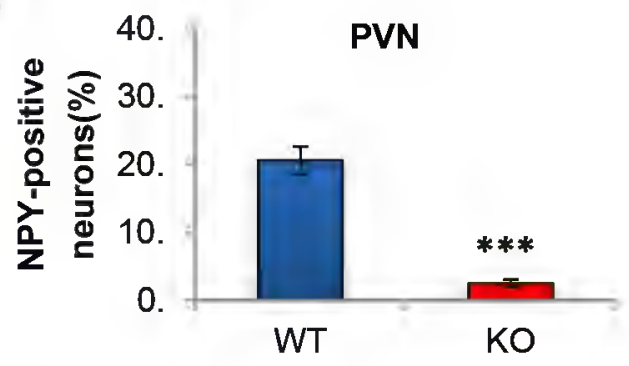

D

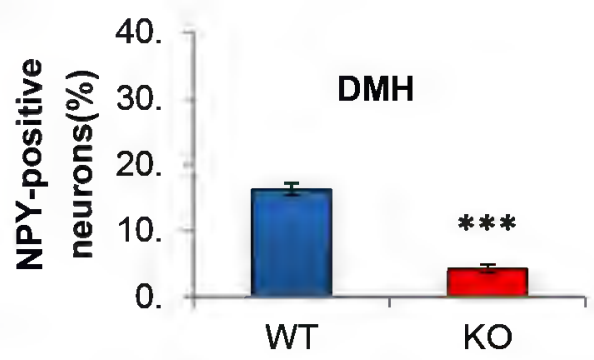

F

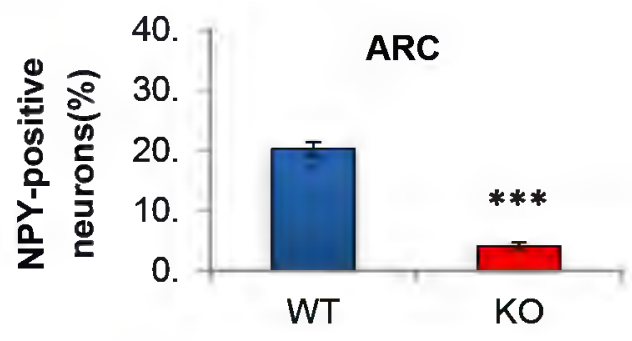

I

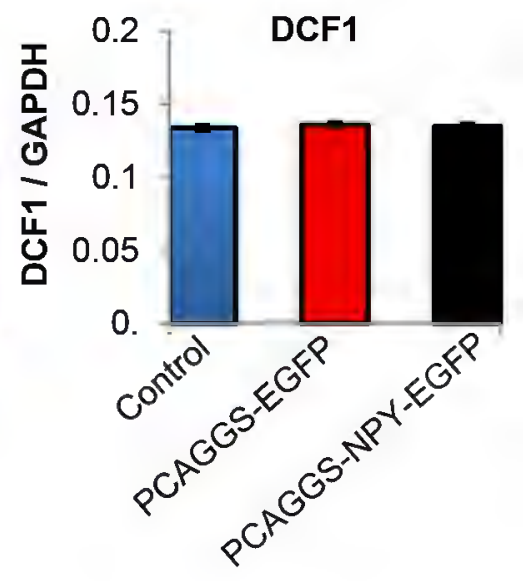


A

B

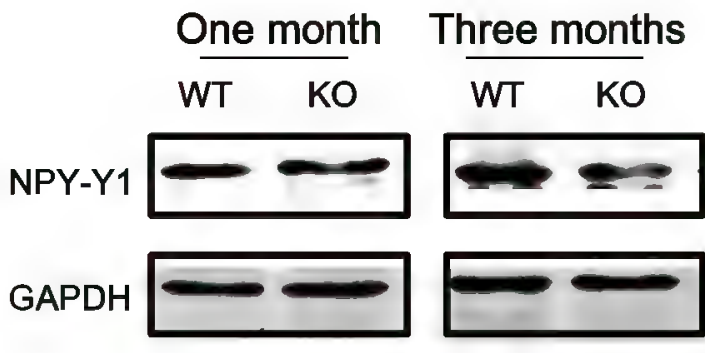

C
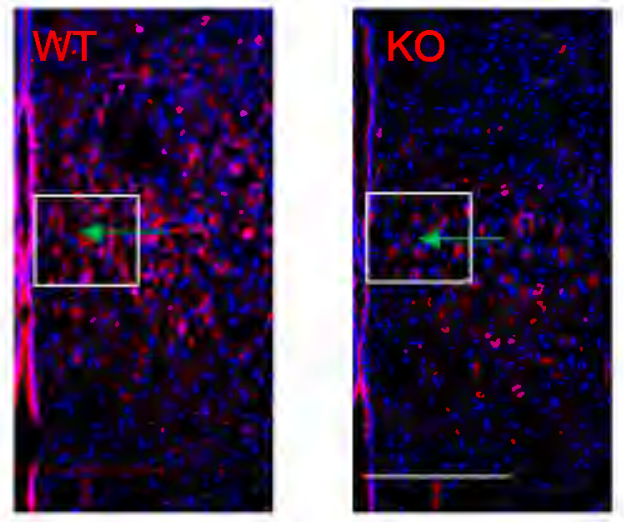

E
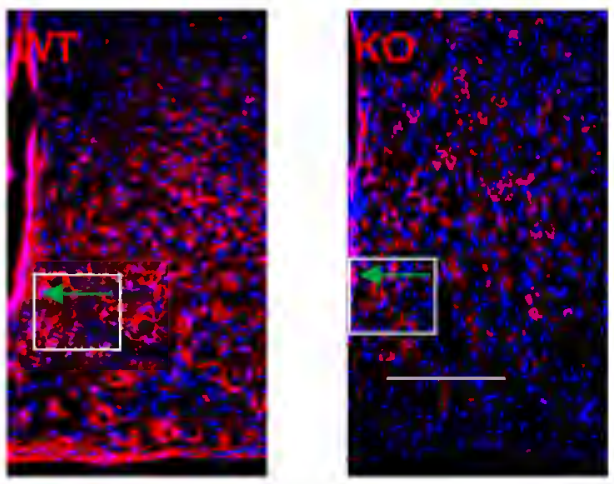

G

PCAGGS PCAGGS -EGFP DCF1-EGFP

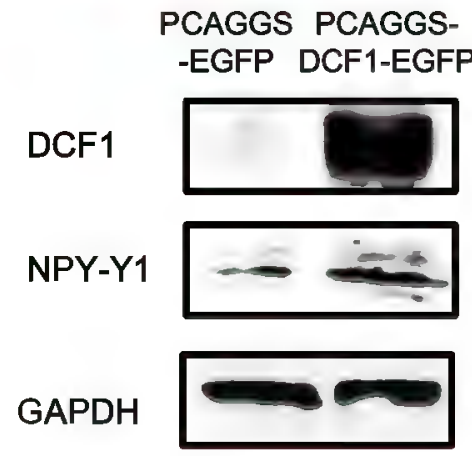

H

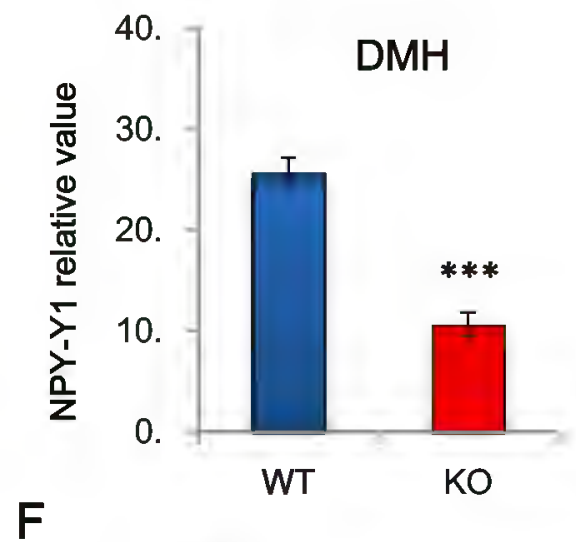

F
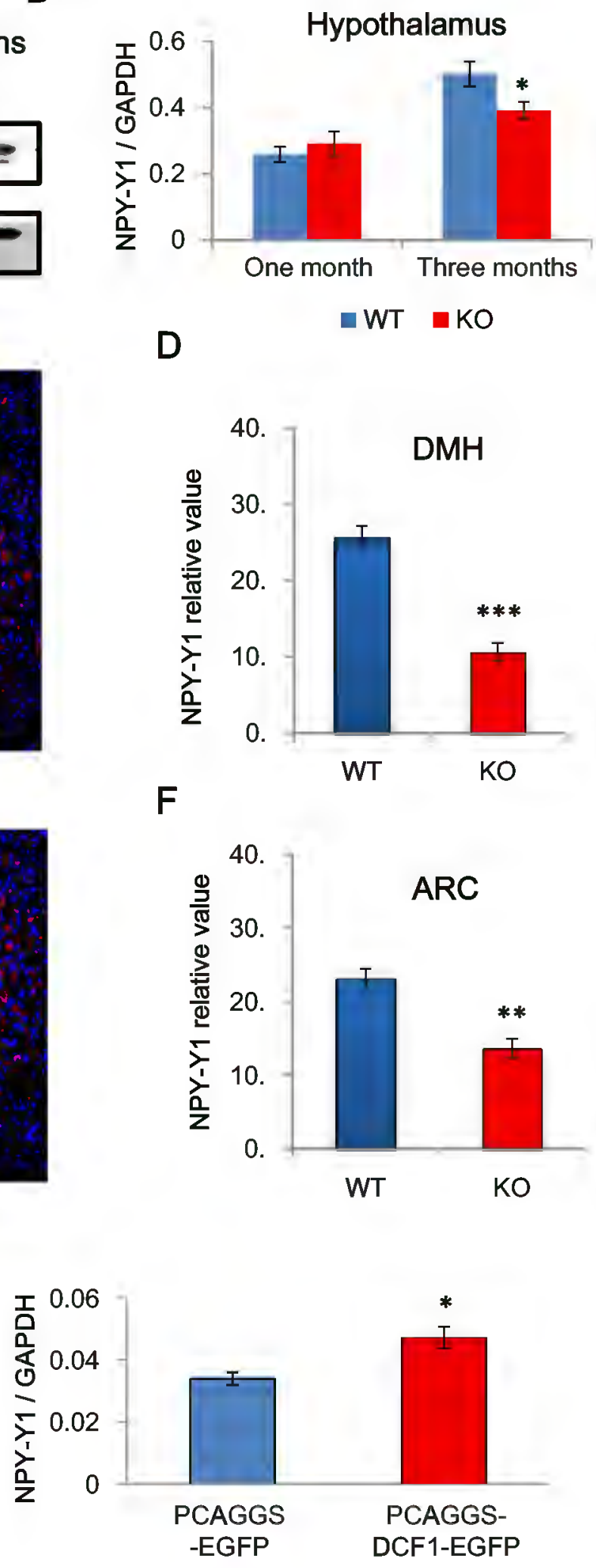
A

B

C
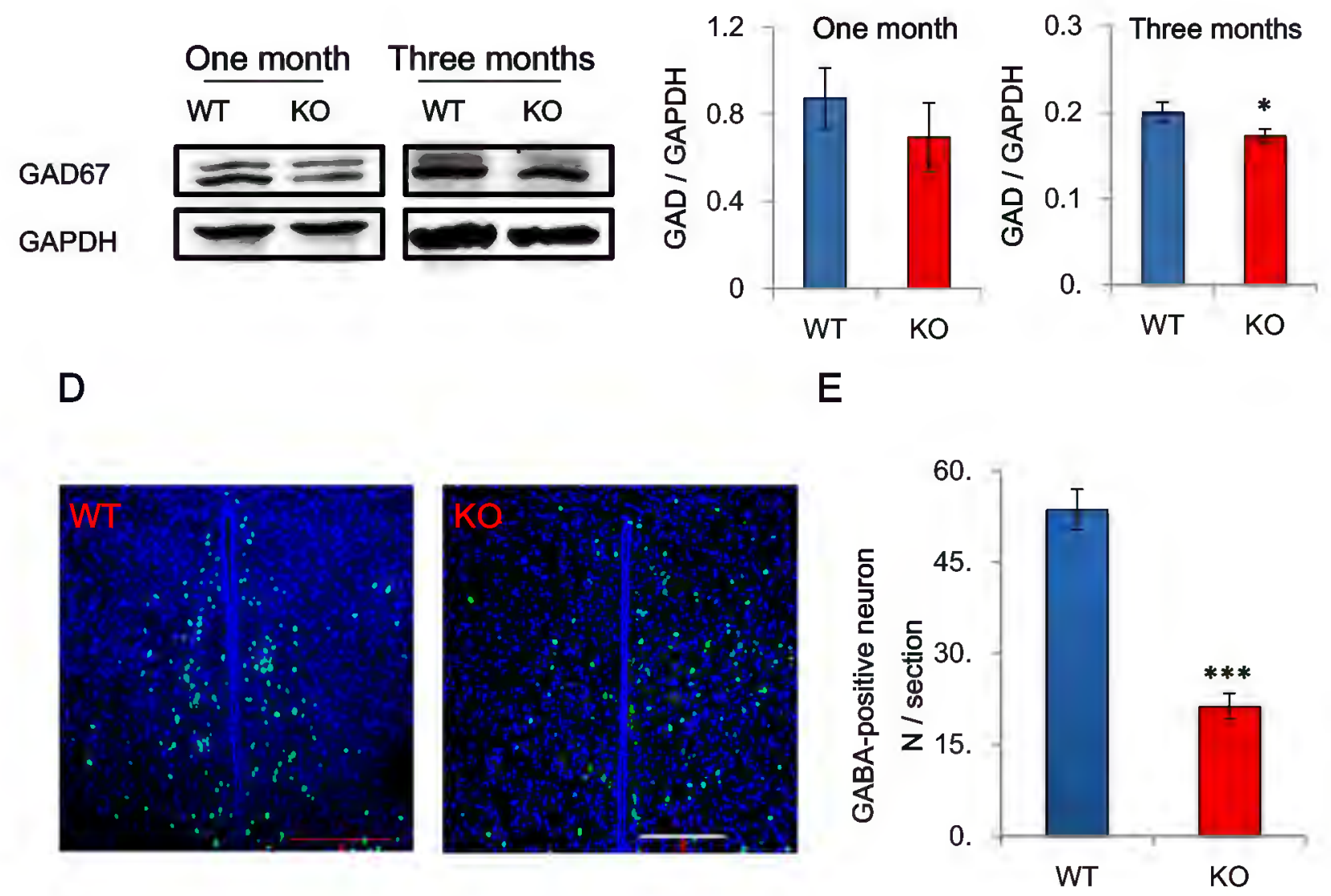

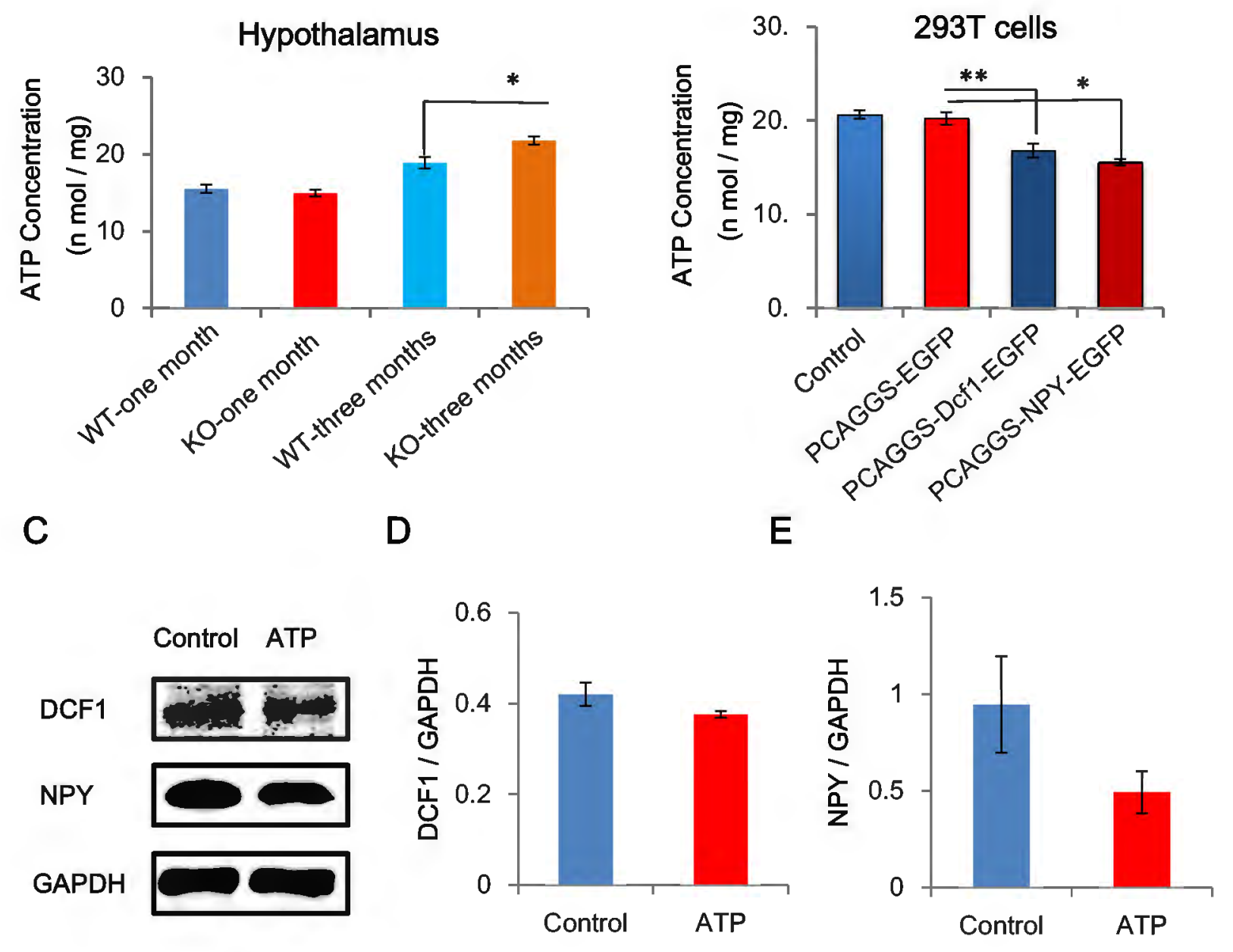